\title{
La Syrie : Terre d'histoire, de civilisations et de guerre
}

Mazigh, $\mathrm{M}^{1 *}$

\section{Résumé}

Alors que des Syriens sont accueillis au Canada, il est utile de savoir d'où ils viennent. La Syrie est une terre ancienne d'une riche histoire et qui a toujours été constituée de cultures, d'ethnicités et de religions diverses. Palmyre était une ancienne civilisation née au Ile siècle. La Syrie est devenue une terre d'Islam en 640 de notre ère et était un centre culturel, artistique et religieux. Au Moyen Âge, la Syrie est tombée sous le contrôle des croisés et a fait partie de l'Empire Ottoman du début des années 1500 jusqu'à la fin du XIXe siècle. Au cours de la Première Guerre mondiale, elle tombe sous l'influence française et est reconnue en tant que nation indépendante après la Deuxième Guerre mondiale.

En 1963, un coup militaire a été mené par Hafez al-Assad, et depuis, la Syrie est régie par l'état d'urgence. Après le décès d'al-Assad en 2000, son fils Bashar al-Assad est élu président dans le cadre d'une campagne présidentielle sans opposition. Avant le conflit actuel, la population de la Syrie était d'environ 22 millions de personnes, mais à présent, près de la moitié de la population a été déplacée dans le pays et dans les pays voisins, y compris environ quatre millions de réfugiés. On estime que 250000 personnes ont trouvé la mort pendant le conflit syrien.

\section{Affiliation}

${ }^{1}$ Coalition pour la surveillance internationale des libertés civiles, Ottawa (ON)

${ }^{\star}$ Correspondance : national. coordination@iclmg.ca

Citation proposée : Mazigh M. La Syrie : Terre d'histoire, de civilizations et de guerre. Relevé des maladies transmissibles au Canada 2016;42-Suppl 2:S1-2. https://doi.org/10.14745/ccdr.v42is2a01f

\section{Introduction}

La Syrie est une terre ancienne d'une riche histoire, qui a toujours été constituée de cultures, d'ethnicités et de religions diverses. Alors que des Syriens sont accueillis au Canada, il est utile de savoir d'où ils viennent.

La Syrie est un pays méditerranéen semi-aride à la géographie diverse. Elle est située à la frontière du Liban, où les plaines sont fertiles; de la Turquie, dans les hautes montagnes du Taurus; de l'Iraq et de la Jordanie, situés dans le désert. Son climat est tempéré en hiver et très chaud et sec en été. Les principales villes de la Syrie sont Damas, Alep, Homs, Lattaquié, Idlib et Raqqa. Pendant I'hiver, il peut neiger pendant quelques jours, mais la neige ne reste jamais longtemps. Le pays est connu pour ses plages magnifiques à Lattaquié et son paysage en haute altitude dans les montagnes du Taurus. La majorité de la population en Syrie vit dans la vallée de l'Euphrate et le long de la plaine littorale, une bande fertile entre les montagnes côtières et le désert.

\section{Diversité culturelle}

La Syrie est un pays diversifié sur le plan culturel dont la population avoisinait les 22 millions d'habitants (1) avant le conflit actuel. La Syrie a toujours été une riche mosaïque composée de diverses ethnies. L'arabe est la langue officielle, mais il existe différents dialectes selon les régions. Le turc, le kurde, l'arménien et l'araméen sont aussi parlés. On pense que l'araméen était la langue parlée par Jésus et ses disciples. Cette langue est toujours parlée par les Assyriens et au cours de cérémonies liturgiques de certains chrétiens de Syrie.

En ce qui a trait à la religion, près des trois quarts de la population sont musulmans, y compris les sunnites, qui constituent le groupe religieux le plus important, et les chiites, comme les ismailis, les duodécimains et les alaouites. Les alaouites contrôlent l'armée, le renseignement et la police et pratiquent la religion de l'actuel président, Bashar al-Assad. Les chrétiens représentent environ $10 \%$ de la population et comprennent les Grecques orthodoxes, les catholiques, les chrétiens syriaques, les chalcédoniens, les antiochiens et les Arméniens orthodoxes. Il y a également les Druzes, les Mandéens et les Yazidis (dont la religion est liée au zoroastrisme) (2). Toutes les fêtes musulmanes et chrétiennes sont des jours fériés en Syrie.

La mosaïque culturelle de la Syrie comprend également de nombreux immigrants et réfugiés. Depuis les années 1800, des personnes venues d'Afghanistan, de Chine, d'Iran et d'Afrique du Nord se sont établies en Syrie. Après la Guerre israélo-arabe de 1948, la Syrie a accueilli plus de 500000 réfugiés palestiniens. En outre, les Iraquiens ont trouvé refuge en Syrie en 2003 pendant la guerre d'Iraq, comme l'ont fait les Libanais en 2006 pendant la guerre israélo-libanaise (2). 


\section{Terre de civilisations anciennes}

Près de 10000 ans avant J.-C., la Syrie était un centre de culture néolithique où l'agriculture, l'élevage de bétail et la poterie ont fait leur apparition pour la première fois dans l'histoire. La ville d'Ebla a été fondée près de 3500 ans avant J.-C. et des fouilles archéologiques ont permis de découvrir des présents de pharaons égyptiens qui suggèrent l'existence de liens commerciaux entre les deux civilisations (2).

La Syrie joue un rôle important et symbolique dans I'histoire de la chrétienté. L'apôtre Paul s'est converti sur le chemin de Damas et est devenu l'une des plus grandes figures de la chrétienté. En Syrie, beaucoup d'églises et de monastères apportent une preuve historique du riche patrimoine chrétien et ancien de la région.

Palmyre était une ancienne civilisation née le long d'une voie commerciale dans une oasis au Ile siècle. C'est un roi de Palmyrène qui vainquit l'empereur persan et qui prit le contrôle de tout l'Empire romain d'Orient, puis, son successeur et la veuve Zénobie établirent l'Empire de Palmyre conquérant brièvement l'Égypte, la Syrie, la Palestine, la majeure partie de l'Asie Mineure, le royaume de Juda et le Liban, avant d'être contrôlé par les Romains en 273 de notre ère.

Malheureusement, le conflit actuel a endommagé beaucoup d'anciens monuments syriens. À l'heure actuelle, les six sites du patrimoine mondial de la Syrie figurent sur la liste des sites menacés (3).

La Syrie est devenue une terre d'Islam en 640 de notre ère. La capitale de la Syrie, Damas (Dimashk ou Al Sham, comme la nomme ses habitants en arabe) a fait partie de la dynastie musulmane des Omeyyades au VII siècle et est devenue un centre culturel, religieux et artistique. La célèbre mosquée des Omeyyades fut construite à cette époque et a attiré de nombreux chercheurs et visiteurs de tout le nouvel empire (4).

\section{Terre de guerre}

Pendant les croisades de 1098 à 1189, les croisés ont pris le contrôle de nombreuses régions syriennes. La Syrie fait ensuite partie de la dynastie Ayyoubide en Égypte.

En 1516, la Syrie est intégrée à l'Empire Ottoman. L'arabe demeure la langue officielle et Damas devient l'escale principale vers la Mecque. Un système social et juridique est élaboré et permet à tous les groupes ethniques de vivre relativement en paix et en harmonie, mais cette paix commence à se déstabiliser au milieu du XIXe siècle, avec l'expansion des empires occidentaux.

À la fin du XIXe siècle, l'Empire Ottoman est soumis de plus en plus à l'influence britannique et française. Au cours de la Première Guerre mondiale, des accords secrets Sykes-Picot sont signés et divisent l'Empire Ottoman en deux zones d'influence britannique et française, et la France reçoit mandat de la Syrie. En 1920, la Syrie et la France négocient un traité d'indépendance qui n'est pas ratifié. Ce n'est qu'en avril 1946 que les Français ont commencé à quitter le pays et que la Syrie a été reconnue par les Nations Unies (1).

Entre les années 1940 et les années 1960, la vie politique syrienne est dominée par les coups et les bouleversements. En 1956, la Syrie signe un pacte avec l'Union soviétique à la suite de la crise de Suez et établit ainsi une influence profonde et durable de la
Russie sur la Syrie par l'intermédiaire d'échanges militaires et du commerce. En 1958, les Syriens s'opposent à une fusion entre l'Égypte et la Syrie.

En 1963, Hafez al-Assad accède au pouvoir grâce à un coup militaire. Depuis, les Syriens vivent sous l'état d'urgence qui a suspendu la constitution et la plupart des droits civils des citoyens. Hafez al-Assad est demeuré au pouvoir de 1970 à 2000, jusqu'à sa mort. Son fils Bashar al-Assad a été élu président dans le cadre d'une campagne présidentielle sans opposition.

\section{Situation actuelle}

On estime que moins de la moitié de la population syrienne ne vit plus chez elle, plus de quatre millions d'habitants sont inscrits comme réfugiés au Liban, en Turquie, en Jordanie, en Iraq et en Égypte (5). Environ 7,6 millions de personnes sont déplacées dans le pays ou vivent dans des camps, des villes ou des régions rurales de pays voisins, mais ne bénéficient pas facilement d'aide humanitaire (5). Depuis le début de la guerre civile, l'économie syrienne a chuté de plus de $60 \%$ et la livre syrienne a perdu plus de $80 \%$ de sa valeur (2). On estime que près de 250000 personnes ont perdu la vie en Syrie au cours du conflit, depuis qu'il a éclaté en mars 2011 (6).

\section{Conflit d'intérêts}

Aucun.

\section{Financement}

Aucun.

\section{Références}

1. United Nations. Economic and Social Affairs. World Statistics Pocketbook, 2014 edition. New York: United Nations; 2014. http://unstats.un.org/unsd/pocketbook/WSPB2014.pdf.

2. Wikipedia contributors. Syria. Wikipedia, the Free Encyclopedia. (Disponible en français : https://fr.wikipedia. org/wiki/Syrie).

3. United Nations Educational Scientific and Cultural Organization (UNESCO). World heritage list. New York: UNESCO; 2015. (Disponible en français : http://whc.unesco. org/fr/list/).

4. Reed FA. Shattered images: The rise of militant iconoclasm in Syria. Vancouver BC: Talonbooks; 2013.

5. Citizenship and Immigration Canada. Population profile: Syrian refugees. Ottawa ON: Government of Canada; November 2015. (Disponible en français : http://www.cpa. ca/docs/File/Cultural/FR\%20Syrian\%20Population\%20Profile. pdf).

6. Aljazeera. 'Almost quarter of a million people' dead in Syria war. Doha, Qatar: Aljazeera; August 7, 2015. http://www. aljazeera.com/news/2015/08/quarter-million-people-deadsyria-war-150807093941704.html. 